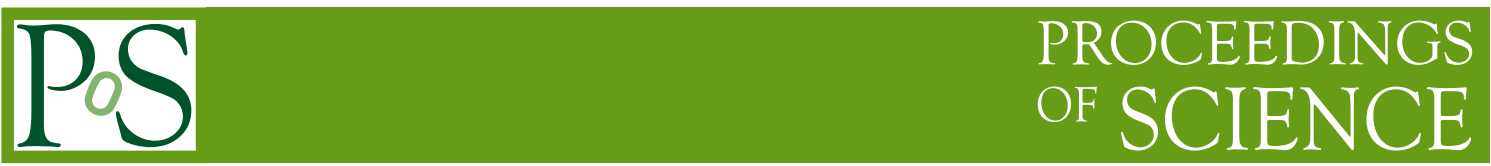

\title{
GEM foil gain prediction
}

\author{
Timo Hildén* \\ Helsinki Institute of Physics, Helsinki, Finland \\ E-mail: timo.hilden@helsinki.fi
}

\section{Jens Erik Brücken}

Helsinki Institute of Physics, Helsinki, Finland

E-mail: erik.brucken@iki.fi

\section{Dezső Varga}

Wigner Research Center for Physics, Budapest, Hungary

E-mail: Varga.Dezso@wigner.mta.hu

\section{Márton Vargyas}

Wigner Research Center for Physics, Budapest, Hungary

E-mail: vargyas.marton@wigner.mta.hu

\begin{abstract}
An optical Quality Assurance (QA) system has been developed at the Detector Laboratory of the Helsinki Institute of Physics. The diameters of the holes in a Gas Electron Multiplier (GEM) -foil, both in the copper and in the polyimide, can be measured with the system. The system is utilized for the QA of the GEM foils of the TPC Read-Out Chambers (ROC) being assembled for the upgrade of the TPC readout chambers of the ALICE experiment at CERN. The correlation between the GEM hole size variation and the corresponding gain variation has been studied with several different gas compositions and operating voltages. A clear correlation has been shown to exist between the size of the GEM holes and the gain of the foil. Furthermore the relative gain of the foil can be estimated within $10 \%$ based solely on the GEM hole size variation. The current correlation results are shown, as well as the progress towards a quantitative prediction of the gain of individual GEM foils. The possibility of predicting the gain of a full GEM stack is discussed. This study has been made in collaboration between Helsinki Institute of Physics and Wigner Research Centre in Budapest as part of the QA effort of the ALICE TPC upgrade project.
\end{abstract}

5th International Conference on Micro-Pattern Gas Detectors (MPGD2017)

22-26 May, 2017

Philadelphia, USA

\footnotetext{
* Speaker.
} 


\section{Introduction}

Previous measurements show a connection between standard Gaseous Electron Multiplier [1] (GEM) foil gain and the diameters of the holes in the foil. A GEM-foil has intrinsic gain variation depending, among other variables, on the variation in hole geometry [2]. This is due to Townsend coefficient being proportional to electric field strength. The exact form of this dependence has, however, remained elusive.

A large area Optical Scanning System (OSS) was developed at the Detector Laboratory of Helsinki Institute of Physics and the Department of Physics of the University of Helsinki. The OSS makes it possible to obtain accurate measurements of the properties of practically every ( $>90$ $\%$ ) individual GEM-hole in a GEM-foil. The development was driven by the needs of the Quality Assurance (QA) procedures of the GEM-foils of the SuperFRS experiment at FAIR and the GEM readout chambers of the upgrade of the TPC of ALICE experiment at CERN.

The ALICE TPC, the largest TPC in the world, is going to be upgraded [3]. In particular the old MultiWire Proportional Chambers (MWPC) are replaced by ReadOut Chambers (ROC) based on GEM technologies. Around $32 \mathrm{~m}^{2}$ of four layer GEM detectors ( $130 \mathrm{~m}^{2}$ of GEM foils) are being assembled for the TPC upgrade. All the foils will be scanned as part of the QA process of the TPC upgrade GEM production [4]. Furthermore the gain of at least one GEM foil per production batch will be measured with a high precision gain scanner in the Wigner Institute at Budapest. Finally each of the 72 ROCs are scanned for gain and Ion Back Flow (IBF) at Bucharest, GSI and Yale. As such, the ALICE TPC upgrade project creates a unique laboratory for studying the basic principles of the formation of gain in GEM detectors.

In the beginning of GEM assembly there has been emphasis on predicting the gain of individual foils, as there is no thorough ROC data ready yet. A comparison of both the geometrical properties and gain performance shows a correlation. A prediction of the performance of the foil is possible with precise information of the shape and size of the holes. However several different measurements can be made, such as the pitch of the holes, the diameter of the hole in the copper and the hole in the polyimide. The objective is to find out how the interplay of these parameters ultimately effects the gain of the GEM foil.

Some parameters cannot be measured with the OSS, such as the depth profile of the holes. There are additionally other factors at play - double pitch foils do not seem to work like the normal foils with 140 micron pitch. Also, preliminary results seem to indicate that when the foils are stacked into a detector the gains of the individual foils combine in a way that is not strictly multiplicative in nature.

\section{Methods}

\subsection{Optical scans}

The optical scanning system (OSS), shown in Fig. 1(a), consists of a custom built robot with modifiable optics and lighting setup as described in [2]. However the setup was changed slightly for the quality assurance of the GEM foils for the new readout chambers of the ALICE Time Projection Chamber. Technical details of the present setup, to accommodate the huge volume of ALICE foils, can be found in [4]. 


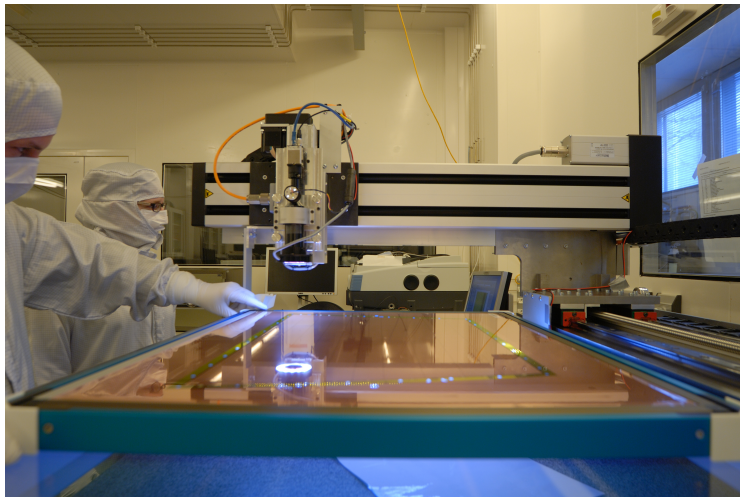

(a)

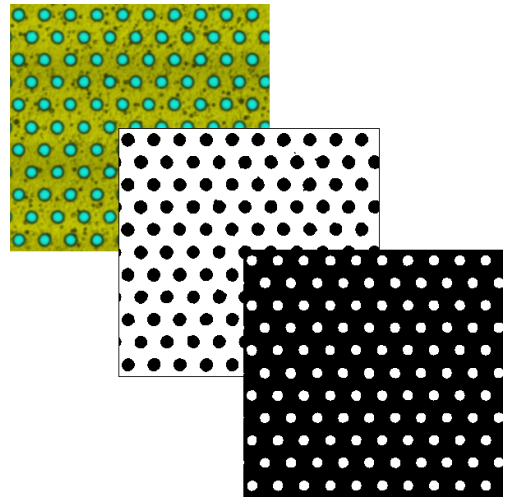

(b)

Figure 1: (a) The high definition optical scanning system. (b) Illustration of the analysis: scanned image, reconstructed copper and polyimide holes.

Originally a $1 \times$ telecentric optics was used with a effective pixel size of 1.75 micron. The magnification was reduced to an effective pixel size of 4.4 micron to increase the scanning speed. To ensure the high quality reconstruction capabilities with lower resolution images a two-exposure mode was introduced with separate exposures for fore- and background-lighting. The so-called two exposure images are false color images composed out of the monochrome images from exposures with the coaxial- and ring-light in the foreground and the back-light.

The basic concept of the reconstruction of geometrical properties of the GEM holes from the scanned foil images has been presented already in [2]. The analysis software was recently upgraded and new features have been developed and added. The properties of the polyimide and copper holes, also often called inner and outer holes, are now reconstructed separately from different exposure channels. This enhances drastically the contrast between outer- and inner holes. The constructed false color image of the two exposures together with the maps of copper and background are shown in Fig. 1(b).

The diameter of the holes are determined by fitting an ellipse to the contour pixels of the holes. This way $20-40$ pixels contribute to the diameter measurement and a sub-pixel resolution can be achieved. It is important, however, to maintain precise control over the lightning conditions for the initial contour recognition to be stable. Due to the high variability in the reflective properties of GEM foils, there is always variation in the surface lighting of different foils. This complicates the measurement of the outer holes. In effect this means that we can measure the relative variation of the diameters of the outer holes within a GEM foil more precisely than the absolute diameter. The achieved resolution for relative diameter measurement of the outer holes is in the order of 1 micron in diameter, whereas the inner holes can be resolved with a resolution of less than 0.5 microns.

The results can be plotted in terms of histograms of maps as show in Fig. 2. Here one can see the maps of the polyimide and copper hole diameters of both sides of the foil.

\subsection{Gain measurement}

At least one GEM foil per GEM production batch is measured in the gain uniformity scanner developed in the Wigner Research Center for Physics. The system was developed to test the gain 


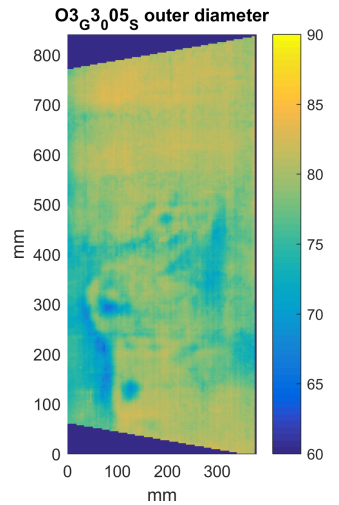

(a)

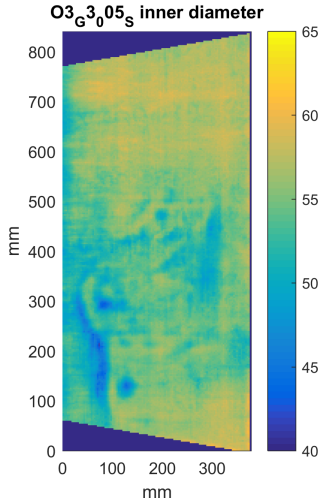

(b)

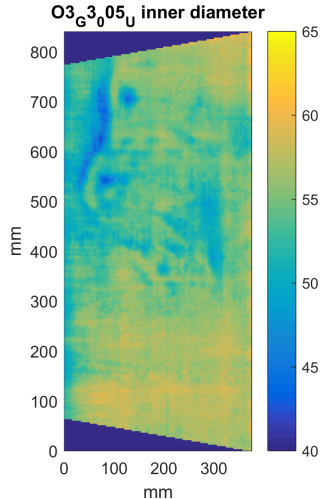

(c)

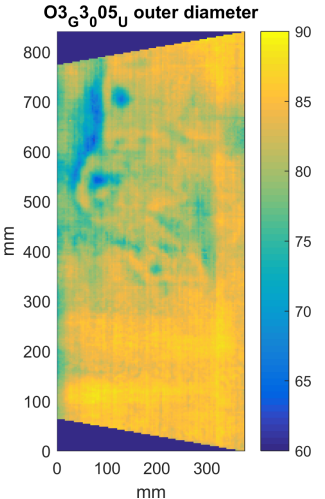

(d)

Figure 2: Diameter maps of a typical ALICE TPC GEM foil, here (a) the copper and (b) the polyimide hole diameters measured from the top side and (c) the polyimide and (d) the copper hole diameters measured from the bottom side of the GEM foil.

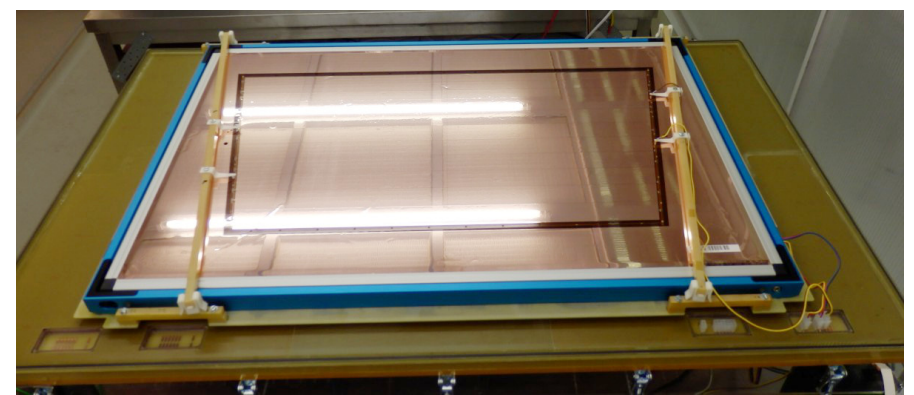

(a)

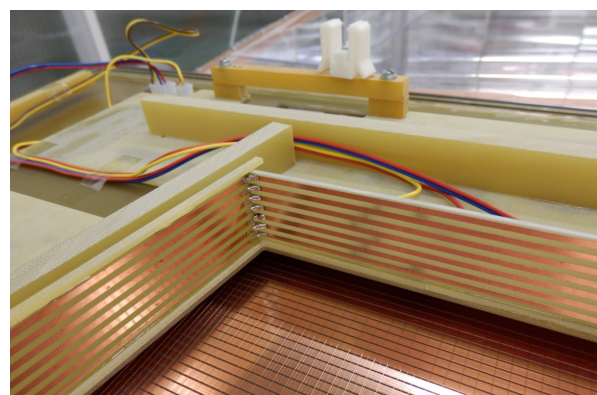

(b)

Figure 3: (a) The The gain mapping system with a mounted GEM foil inside. (b) A closeup of the the system without GEM foil showing the underlying MWPC and field cage.

uniformity of the ALICE TPC upgrade GEM foils as part of the QA protocol. The device is shown in Fig. 3(a) with a test GEM already mounted in open stage. It consists of a MWPC with y-strip readout. This is visible in Fig. 3(b) that also shows the field cage to limit field non-uniformity around the borders. It is designed large enough to fit the largest GEM foils used for the ALICE TPC $(>80 \mathrm{~cm})$. The measurement resolution is $4 \mathrm{~mm} \times 3 \mathrm{~mm}$. The device is carefully controlled during measurements to obtain maximum repeatability.

The achievable accuracy is close to $5 \%$, which is remarkable taking into account the large gas volume of the device, the rather large transfer gap between the GEM and the MWPC and the fact that inexpensive industrial gas mixture of Argon and $\mathrm{CO}_{2}$ is used as a fill gas. After thorough flushing with the gas mixture the device is irradiated from above by a ${ }^{55} \mathrm{Fe} \mathrm{X}$-ray source with a rate of approximately $3 \mathrm{kHz}$. The drift field is setup to 300 (400) V/cm and the transfer field to 500 $\mathrm{V} / \mathrm{cm}$ with 330 (320) V over the foil for standard pitch (large pitch) foils. Foils were run with the effective gain of 5 for standard pitch foils and 8 for large pitch foils. Fig. 4 shows as a result a gain map of an ALICE GEM foil. 


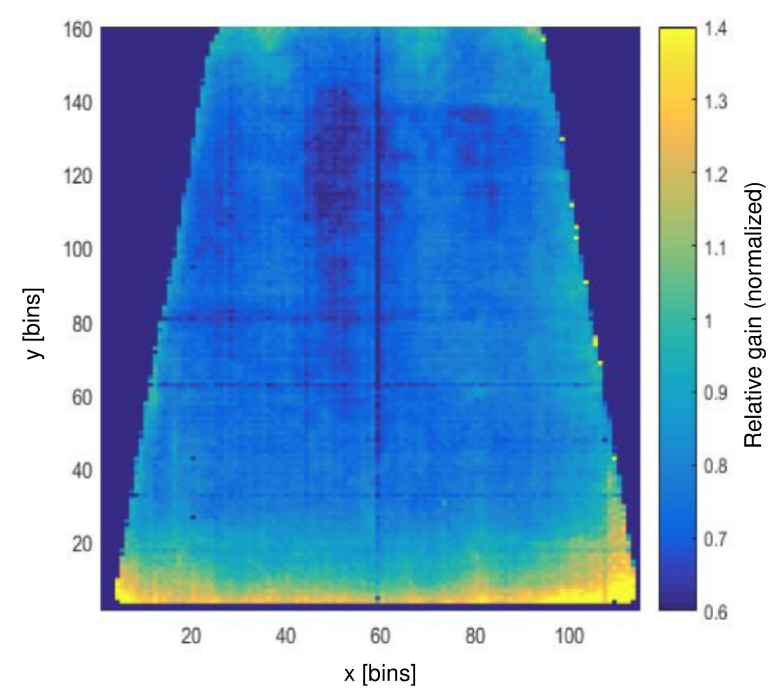

Figure 4: A map of relative gain of a typical ALICE TPC GEM foil.
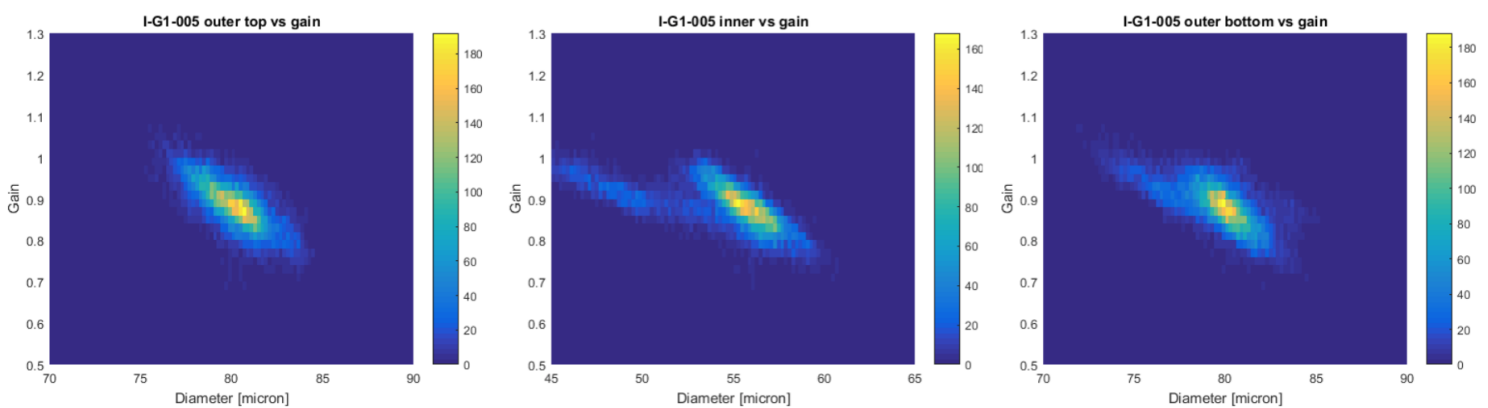

Figure 5: Comparison of single hole properties with gain of a standard pitch ALICE GEM foil.

\section{Results}

By comparing the maps of the geometrical hole properties with the gain maps one can see immediate correlation even without going into details. Fig. 5 shows the comparison of the polyimide and copper holes with the local gain of an ALICE GEM foil. An inverse correlation between hole diameters and gain is evident from the plots. In Fig. 6 the gain of 4 different foils is plotted as a function of the average diameter of copper holes measured from both sides of the foils. We can conclude that there is a inverse linear dependency between the outer hole diameter and the logarithm of the gain.

The above results seem unambiguous, but when the process was repeated with foils with twice the pitch of the hole pattern ( 280 microns) the results did not fall into the same pattern as with the standard pitch ones. The reason of this discrepancy is not understood at the moment. Fig. 7 shows the comparison of the polyimide and copper holes with the local gain of a double pitch ALICE GEM foil. 


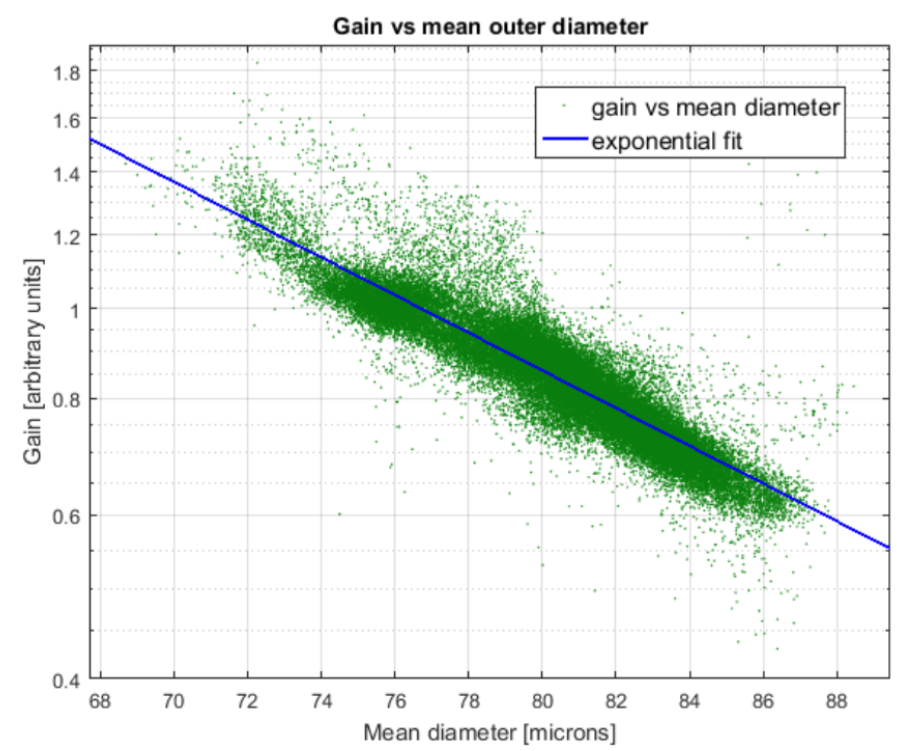

Figure 6: Comparison of averaged top and bottom copper holes to the gain of four foils.
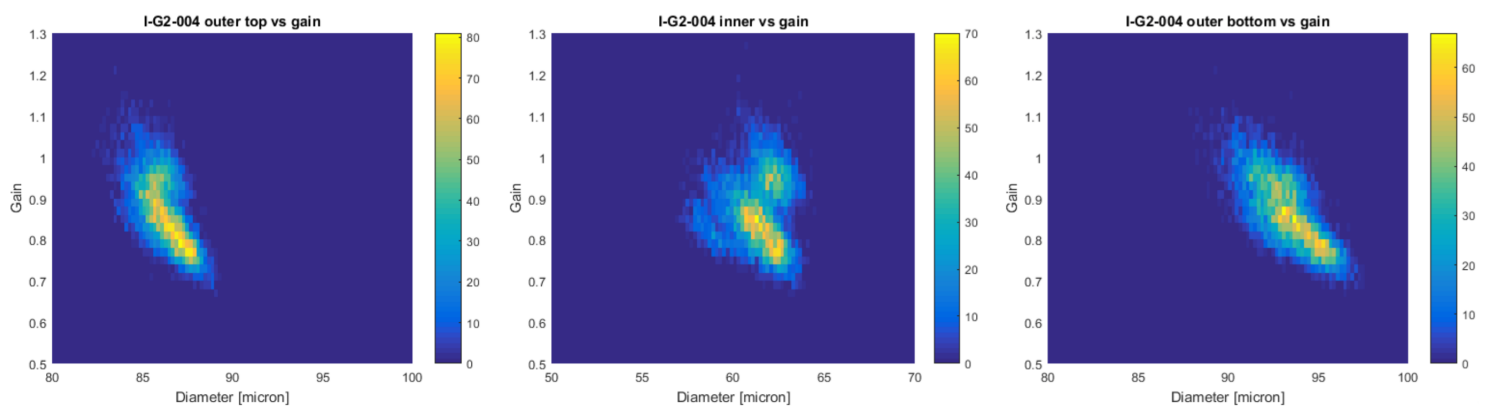

Figure 7: Comparison of single hole properties with gain of a double pitch ALICE GEM foil.

\section{Summary}

We studied the correlation of hole-geometries to the gain of single mask GEM foils. A clear correlation has been found for standard pitch foils. For double pitch foils, which are also used in the ALICE TPC upgrade ROCs, the correlation is somewhat more complicated. The gain does not depend on the outer hole diameter variation in the same way as the gain of the standard pitch foils does.

The goal is to predict the gain of a full GEM stack with a field setup as used for the ALICE TPC upgrade ROCs. This is not trivial and according to preliminary results simple multiplication of the gain of individual foils does not predict the gain of a full stack. Single foil gain variance seems to always be worse than the combined variance of the detector gain. To shed light on this problem the study of the ALICE TPC ROC as a dynamic system with very specific field setup is needed. 


\section{References}

[1] F. Sauli, GEM: A new concept for electron amplification in gas detectors, Nucl. Instr. Meth. A 386, (1997) 531.

[2] T. Hildén, E. Brücken, J. Heino, M. Kalliokoski, A. Karadzhinova, R. Lauhakangas, E. Tuominen and R. Turpeinen, Optical quality assurance of GEM foils, Nucl. Instr. Meth. A 770 (2014) 113.

[3] The ALICE Collaboration, Technical Design Report for the Upgrade of the ALICE Time Projection Chamber, CERN- LHCC-2013-020 (2014).

[4] E. Brücken and T. Hildén, The GEM QA Protocol of the ALICE TPC Upgrade Project, submitted to POS (2018) (these proceedings). 